\title{
Studies of the Verwey Transition in Magnetite
}

\author{
Z. Tarnawski*, A. Wiecheć, M. Madej, D. Nowak, \\ D. Owoc, G. Król, Z. Ka̧Kol, L. Kolwicz-ChOdak, \\ A. KozŁowski And T. DAwid \\ Department of Solid State Physics \\ Faculty of Physics and Applied Computer Science \\ AGH University of Science and Technology \\ al. Mickiewicza 30, 30-059 Kraków, Poland
}

\begin{abstract}
Studies of the specific heat and simultaneous AC magnetic susceptibility $\left(\chi^{\prime}\right)$ and electric resistance of stoichiometric magnetite single crystal are presented. The temperature hysteresis of the Verwey transition is of $0.03 \mathrm{~K}$ found from the specific heat data confirming its first-order character. The continuous temporal change of $\chi^{\prime}$ at $T_{\mathrm{V}}$ can be switched off by an external magnetic field without affecting the transition. The electrical resistance decreases continuously with increasing temperature with a rapid change of slope at the point when the phase transition is completed. It was concluded that the magnetic degrees of freedom do not actively participate in the transition and that the entropy released at $T_{\mathrm{V}}$ may come from ordering electrons.
\end{abstract}

PACS numbers: 61.18.Bn, 75.40.-s, 71.20.Lp

\section{Introduction}

Magnetite is a mixed valence compound in which the iron ionic pattern at the octahedral sites is usually believed to change at the Verwey transition temperature $T_{\mathrm{V}}$ of $125 \mathrm{~K}$ from a long-range low-temperature order (exhibiting very limited electrical conduction) to a high-temperature dynamic disorder $[1,2]$. In the high-temperature phase, the "additional" electrons of the $\mathrm{Fe}^{2+}$ ions were considered to resonate between adjacent octahedral $\mathrm{Fe}^{3+}$ positions implying a drastic increase in

*corresponding author; e-mail: tarnawsk@uci.agh.edu.pl 
electrical conduction. The Verwey transition is also reflected by anomalies in many other physical properties, such as a jump in the magnetic susceptibility, a sharp peak in the specific heat and a change of the crystal symmetry from monoclinic (space group $C c$ ) below $T_{\mathrm{V}}$ to cubic $(F d \overline{3} m)$ above $T_{\mathrm{V}}$. Despite of extensive studies the main mechanisms responsible for the Verwey transition are still unclear. Especially such an order-disorder transition, which was well established for over 60 years, is now heavily questioned [3, 4]. Thus the detailed description of the Verwey transition becomes again more vital.

In our study we focused on a delivery of the heat of the transition to the sample exactly at the transition temperature, i.e. we traced the transition in statu nascendi. In particular, we aimed at resolving the transition hysteresis. The Verwey transition was always considered of the first order and the hysteresis have not been observed until now. Again remaining exactly at the transition temperature, we simultaneously measured temporal dependence of electrical resistance and magnetic susceptibility.

\section{Experimental results and discussions}

The single crystalline magnetite $\mathrm{Fe}_{3} \mathrm{O}_{4}$ was prepared from $99.99 \%$ pure $\mathrm{Fe}_{2} \mathrm{O}_{3}$ powder by the skull melter technique. It was then annealed in appropriated conditions established for obtaining a stoichiometric sample. The specific-heat measurements was performed on a home-made setup using the quasi-adiabatic method. AC susceptibility was measured on a commercial LakeShore ACS7000 series susceptometers in a frequency of $188.88 \mathrm{~Hz}$. The electrical resistance was measured by a 2-points silver-paint contact.

The temperature dependence of specific heat $\left(C_{p}\right)$, electrical resistance $(R)$ and AC susceptibility $\left(\chi^{\prime}\right)$ for the stoichiometric samples of magnetite single crystal were shown in Fig. 1. In all cases a discontinuity at $T_{\mathrm{V}}$ was observed. The entropy released at the Verwey transition is $6.4 \mathrm{~J} /(\mathrm{mol} \mathrm{K})$. The temporal profile of the calorimeter temperature on heating and cooling in a quasi-adiabatic condition is displayed in Fig. 2. Utilizing two relaxation time model of heat flow to the sample holder and sample itself, the temporal changes of sample temperature were evaluated. The inverse time derivative of sample temperature revealed distinct peaks at $123.28 \mathrm{~K}$ and $123.31 \mathrm{~K}$, respectively. In both cases the transition width is of about $0.03 \mathrm{~K}$. Moreover, a hysteresis of $0.03(5) \mathrm{K}$ was observed. We notice here that the temperature difference between the sample and calorimeter thermometer was less than $3 \mathrm{mK}$, i.e. one range lower than the observed hysteresis. The temporal variation of sample temperature and AC susceptibility in zero DC magnetic field and in an applied field of $2 \mathrm{kOe}$ during heating across the transition are presented in Fig. 3. In zero field a gradual rearrangement of the magnetic moments was observed until the transition is completed. In the applied field $\chi^{\prime}$ is largely diminished. Moreover, no indication of the transition was found. Thus, the 


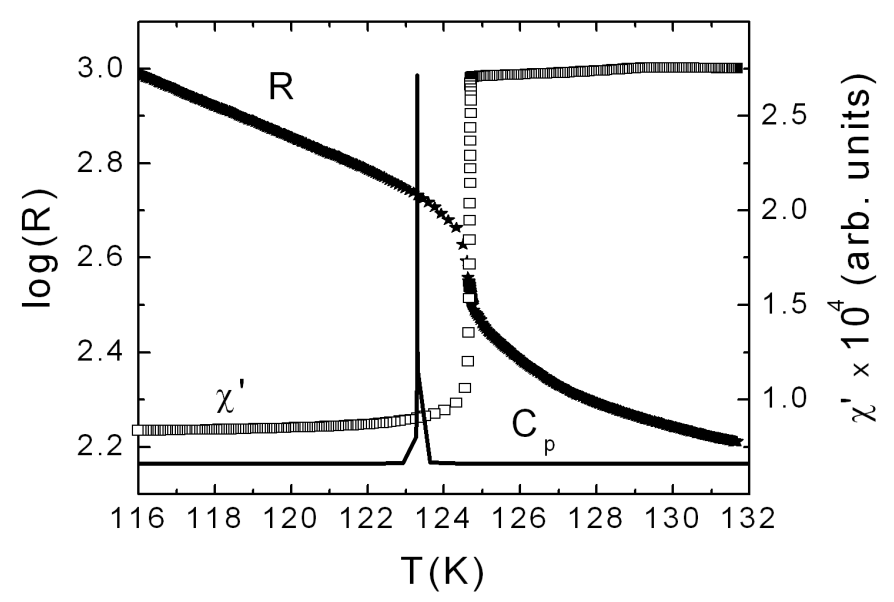

Fig. 1. The temperature dependence of specific heat $\left(C_{p}\right)$, electrical resistance $(R)$ and susceptibility $\left(\chi^{\prime}\right)$ in the phase transition region. An arbitrary unit (not displayed in the figure) was used for $C_{p}$. For clarity the temperature scale for $R$ and $\chi^{\prime}$ was shifted with respect to the true temperature scale.

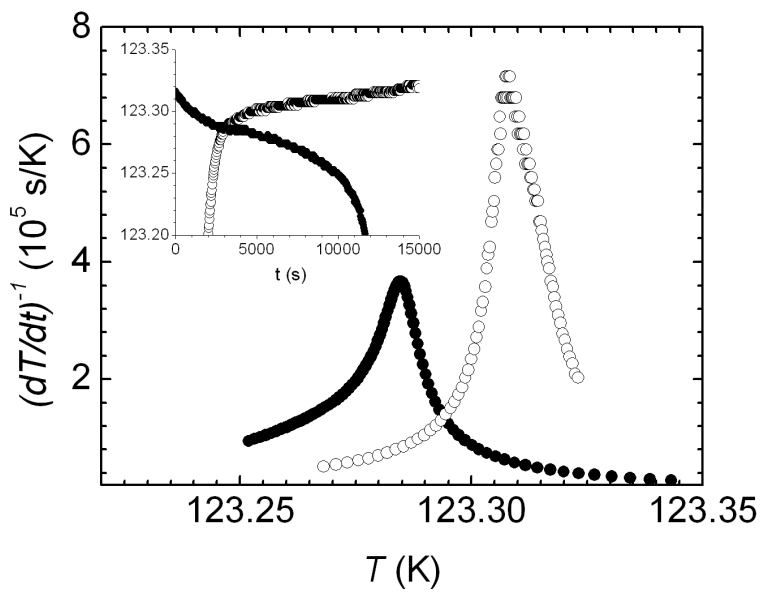

Fig. 2. The inverse time derivative of temperature on warming (o) and cooling (•) of the sample in a quasi-adiabatic condition. Insert: the time dependence of sample temperature.

jump at the transition in the susceptibility (see Fig. 1) is a side effect related to a change in magnetic domain wall movement coupled to either the crystal-structure change or electron order-disorder transformation at $T_{\mathrm{V}}$. Figure 4 showed the data of the temporal variation of sample temperature and electrical resistance on heating and cooling across the transition. The temporal variation of the resistance has a jump at the beginning of the transition and then decreases gradually with time until transition is completed. However, the temperature dependence of the 


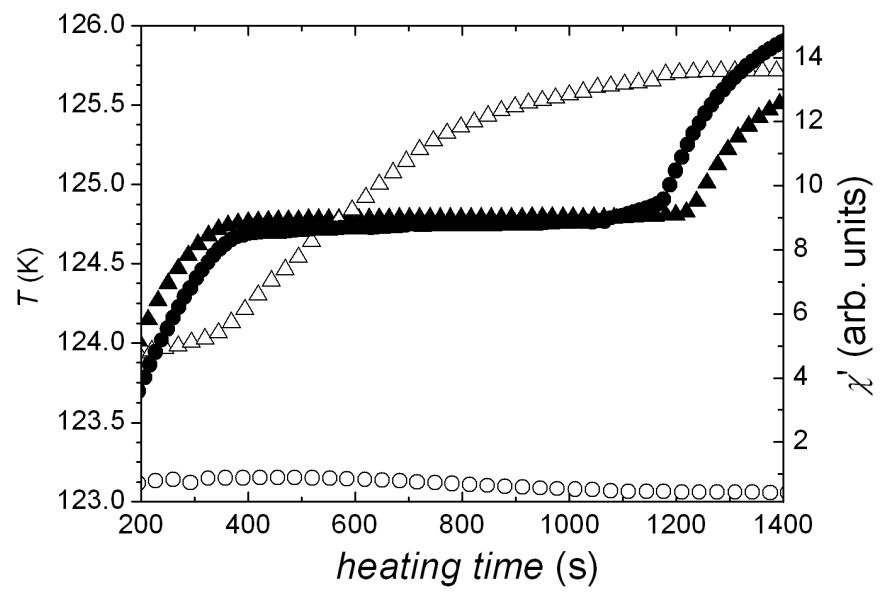

Fig. 3. The temporal variation of sample temperature (full triangle, $\bullet$ ) and AC susceptibility $(\triangle, \circ)$. Closed markers showed the data in zero field. Open markers in applied external magnetic field of $2 \mathrm{kOe}$.

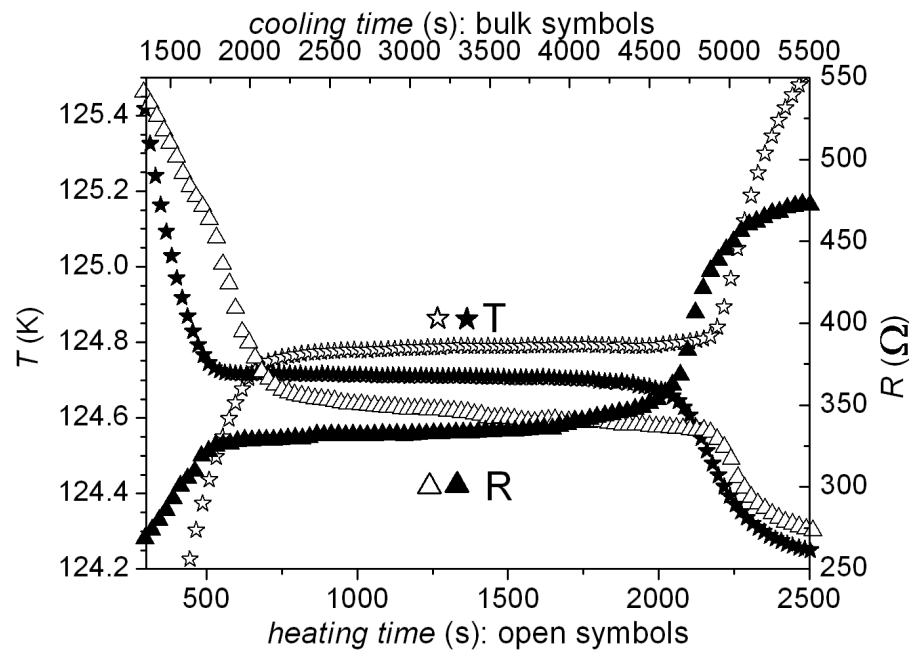

Fig. 4. The temporal dependence of sample temperature $(T)$ and electrical resistance $(R)$ on heating (solid markers) and cooling (open markers) through the transition.

resistance shows a continuous decrease with increasing temperature in the vicinity of the transition with no characteristic feature at the beginning of the transition. But a drop with a rapid change of slope afterwards is observed at the end of the transition.

Our observation is in a qualitative agreement with the concept of melting electronic pattern linked to the lattice [5]. However the large entropy gain at the transition indicated that not only electronic degrees of freedom, but maybe also the 
rearrangement of the lattice was involved. Thus the time-dependence investigation of the structure change is highly needed in order to observe the proliferation of the new high symmetry crystallographic phase.

\section{Summary}

The time-dependence investigations of the Verwey transition process showed an existence of a hysteresis of $0.03 \mathrm{~K}$ at $T_{\mathrm{V}}$. From the $\chi^{\prime}$ measurements it was found that the transition is not driven by the magnetic interactions despite of the observed discontinuity in $\chi^{\prime}$ at $T_{\mathrm{V}}$. The electrical resistance changes can be understood within the phase separation model with a decrease in volume of the low-temperature and high-resistance phase. However, an opening of the gap (with decreasing temperature) cannot be excluded. To the best of our knowledge, it was the first experiment revealing a hysteresis of the Verwey transition of the stoichiometric magnetite.

\section{Acknowledgments}

This work is financially supported by the State Committee for Scientific Research.

\section{References}

[1] E.J. Verwey, Nature (London) 144, 327 (1939).

[2] F. Walz, J. Phys., Condens. Matter 14, R285 (2002).

[3] J. Garcia, G. Subias, J. Phys., Condens. Matter 16, R145 (2004).

[4] J.P. Wright, J.P. Attfield, P.G. Radealli, Phys. Rev. B 66, 214422 (2002).

[5] J.M. Honig, J. Spałek, J. Solid State Chem. 90, 115 (1992). 